\title{
Modules that detect finite homological dimensions
}

\author{
Olgur Celikbas, Hailong Dao, and Ryo Takahashi
}

\begin{abstract}
We study homological properties of test modules that are, in principle, modules that detect finite homological dimensions. The main outcome of our results is a generalization of a classical theorem of Auslander and Bridger: we prove that if a commutative Noetherian complete local ring $R$ admits a test module of finite Gorenstein dimension, then $R$ is Gorenstein.
\end{abstract}

\section{Introduction}

Throughout this paper, we assume that all rings are commutative Noetherian rings and all modules are finitely generated. Unless otherwise specified, $R$ denotes a local ring with maximal ideal $\mathfrak{m}$ and residue field $k$. The aim of this paper is to study test modules.

\section{DEFINITION 1.1}

An $R$-module $M$ is called a test module if all $R$-modules $N$ with $\operatorname{Tor}_{\gg 0}^{R}(M, N)=0$ have finite projective dimension.

There are many examples of test modules with interesting consequences in the literature. For instance, it is well known that the residue field $k$ of $R$ is a test module (see [9, Section 1.3]). In general it requires highly nontrivial results to characterize all test modules, even over specific rings. One such result is due to Huneke and Wiegand [20, Theorem 1.9]: a test module over a singular hypersurface is nothing but a module of infinite projective dimension. This result was later obtained by Miller (see proof of [28, Theorem 1.1] in Section 1) and the third author [31, Corollary 7.2] by using different techniques.

In this paper we consider test modules discussed above in a broader context by studying their homological properties. We investigate when a module-finite algebra is a test module, and we prove the following.

Kyoto Journal of Mathematics, Vol. 54, No. 2 (2014), 295-310

DOI 10.1215/21562261-2642404, (C) 2014 by Kyoto University

Received July 24, 2012. Accepted January 31, 2013.

2010 Mathematics Subject Classification: Primary 13D07; Secondary 13D05, 13H10.

Dao's work partially supported by National Science Foundation grants DMS 0834050 and DMS 1104017.

Takahashi's work partially supported by Japan Society for the Promotion of Science Grant-in-Aid for Young Scientists (B) 22740008 and by Japan Society for the Promotion of Science Postdoctoral Fellowships for Research Abroad. 


\section{THEOREM 1.2}

Let $R \rightarrow S$ be a finite local homomorphism of local rings. Assume that either

(1) the ring $S$ is regular or

(2) there exists a test $S$-module that has finite projective dimension over $R$.

Then $S$ is a test $R$-module.

It is remarkable that the existence of a test module of finite homological dimension characterizes the ring itself: if there exists a test module of finite projective dimension, then $R$ is regular (see [9, Theorem 2.2.7]). Auslander and Bridger [3] proved that if the residue field $k$ has finite $G$-dimension (see Definition 3.1), then $R$ is Gorenstein. Corso-Huneke-Katz-Vasconcelos and Goto-Hayasaka, under mild technical conditions, generalized this classical theorem: Let $I$ be an integrally closed $\mathfrak{m}$-primary ideal of a local ring $R$. Then $I$ is a test module (see $[14$, Corollary 3.3]). If $I$ has finite G-dimension and contains a non-zero-divisor of $R$, then $R$ is Gorenstein (see [17, Theorem 1.1(2)]). We will obtain a generalization in this direction.

\section{THEOREM 1.3}

Let $R$ be a homomorphic image of a Gorenstein local ring. If there exists a test module of finite G-dimension, then $R$ is Gorenstein.

We also study the structure of test and nontest modules over complete intersections. Recall that $R$ is called a complete intersection (resp., hypersurface) if its completion is a quotient of a regular local ring by a regular sequence (resp., regular element). Auslander and Bridger [3] introduced the notion of a resolving subcategory of the category $\bmod R$ of finitely generated $R$-modules, which is a full subcategory containing free modules and closed under direct summands, extensions, and syzygies. For each $R$-module $M$ the smallest resolving subcategory containing $M$ is called the resolving closure of $M$. We will prove the following.

\section{THEOREM 1.4}

Let $R$ be a complete intersection.

(1) The test $R$-modules are precisely the $R$-modules of maximal complexity.

(2) The nontest $R$-modules form a resolving subcategory of $\bmod R$. If it is written as the resolving closure of some module, then $R$ is a hypersurface.

The first assertion extends the result of Huneke and Wiegand stated above. The second says that nontest modules form a good subcategory but its structure is not simple in general.

The organization of this paper is as follows. In Section 2, we analyze basic properties of test modules. Theorem 1.2(1) and an extended version of Theorem 1.2(2) are shown in this section (Proposition 2.4 and Theorem 2.5). We also prove Theorem 1.4(1) in this section (Proposition 2.7). In Section 3, we study 
the existence of test modules of finite homological dimensions. We characterize test modules in terms of the vanishing of Ext, which yields a generalized version of Theorem 1.3 (Theorem 3.2). In Section 4, we develop categorical approaches for nontest modules. Theorem $1.4(2)$ is proved in this section (Corollaries 4.4 and 4.10).

\section{Basic properties of test modules}

In this section we analyze basic properties of test modules. We should note that modules akin to test modules were studied in the literature before (see, e.g., [21] and $[27])$.

First of all, we remark that our definition of a test module is different from the one defined by Ramras [29, Lemma 1.1] (see also [1] and [25]). He defined and studied test modules for projectivity in terms of the vanishing of a single Ext module. More precisely, an $R$-module $M$ is called an Ext-test module (a test module in the sense of [25] and [29]) if every $R$-module $P$ with $\operatorname{Ext}_{R}^{1}(P, M)=0$ is free. We record a few observations concerned with the test and Ext-test modules.

\section{REMARK 2.1}

(1) Nontrivial examples of test modules over arbitrary local rings are abundant: if $(R, \mathfrak{m})$ is a local ring and $M \in \bmod R$, then there exists an integer $n>0$ such that $\mathfrak{m}^{n} M$ is a test module (see [2, 1.5(1), Definition 2.1, Propositions 2.2, and 2.3(5)] and [33, Lemma 2.4(b)].

(2) Test and Ext-test modules are different in general: by definition an Exttest module has depth at most one, but a test module does not necessarily have this depth restriction.

(3) Ext-test modules are indeed test modules over complete intersections: this follows from the fact that the vanishing of $\operatorname{Ext}^{\gg 0}(-,-)$ is equivalent to the vanishing of $\operatorname{Tor}_{\gg 0}(-,-)$ over complete intersections by [5, Theorem 6.1$]$.

(4) Test modules are indeed Ext-test modules over hypersurfaces that are either Artinian rings or one-dimensional domains: Assume that $R$ is such a ring, assume that $M$ is a test $R$-module, and assume that $\operatorname{Ext}_{R}^{1}(P, M)=0$ for some $P \in \bmod R$. We may assume by [25, Theorem 1] that $R$ is nonregular, whence $\operatorname{pd}_{R} M=\infty$. We see that $\operatorname{Ext}_{R}^{>0}(P, M)=0$ from [8, Corollary 3.5] and [10, Corollary 4.14]. This implies that $P$ is free by $[5,5.12]$.

Next we prove that test modules behave well modulo non-zero-divisors. We denote by $\mathrm{T}(R)$ the full subcategory of $\bmod R$ consisting of test modules, and by $\Omega^{n} M$ (or $\Omega_{R}^{n} M$ when necessary) the $n$th syzygy of an $R$-module $M$.

PROPOSITION 2.2

Let $(R, \mathfrak{m})$ be a local ring, and let $M$ be an $R$-module. Let $x \in \mathfrak{m}$ be a non-zerodivisor on $M$. Then:

(i) $M \in \mathrm{T}(R)$ if and only if $M / x M \in \mathrm{T}(R)$.

(ii) Assume further that $x$ is a non-zero-divisor on $R$. 
(a) If $M / x M \in \mathrm{T}(R / x R)$, then $M \in \mathrm{T}(R)$.

(b) If $x \notin \mathfrak{m}^{2}$ and $M \in \mathrm{T}(R)$, then $M / x M \in \mathrm{T}(R / x R)$.

Proof

There is an exact sequence $0 \rightarrow M \stackrel{x}{\rightarrow} M \rightarrow M / x M \rightarrow 0$. Hence, $\operatorname{Tor}_{\gg 0}^{R}(M, N)=0$ if and only if $\operatorname{Tor}_{\gg 0}^{R}(M / x M, N)=0$ for all $R$-modules $N$. This proves (i). For the rest of the proof, we assume that $x$ is a non-zero-divisor on both $R$ and $M$. Assume that $M / x M \in \mathrm{T}(R / x R)$, and assume that $\operatorname{Tor}_{\gg 0}^{R}(M, N)=0$ for some $R$-module $N$. Then, by the above exact sequence, $\operatorname{Tor}_{\gg 0}^{R}\left(M / x M, N^{\prime}\right)=0$, where $N^{\prime}:=\Omega N$. Then, since $x$ is a non-zero-divisor on both $R$ and $N^{\prime}$, it follows that $\operatorname{Tor}_{\gg 0}^{R / x R}\left(M / x M, N^{\prime} / x N^{\prime}\right)=0$. Therefore, $\operatorname{pd}_{R / x R}\left(N^{\prime} / x N^{\prime}\right)<\infty . \operatorname{As}_{\operatorname{pd}_{R}}\left(N^{\prime}\right)=$ $\operatorname{pd}_{R / x R}\left(N^{\prime} / x N^{\prime}\right)$ by [9, Lemma 1.3.5], this proves (ii)(a). Finally, assume that $x \notin \mathfrak{m}^{2}$ and $M \in \mathrm{T}(R)$. Suppose that $\operatorname{Tor}_{\gg 0}^{R / x R}(M / x M, T)=0$ for some $R / x R$ module $T$. Then, as $\operatorname{Tor}_{i}^{R / x R}(M / x M, T) \cong \operatorname{Tor}_{i}^{R}(M, T)$ for all $i \geq 0$, we have $\operatorname{pd}_{R} T<\infty$. Since $x \notin \mathfrak{m}^{2}$, we have $\operatorname{pd}_{R / x R} T<\infty$ by [4, Proposition 3.3.5(1)]. This proves that $M / x M \in \mathrm{T}(R / x R)$ and hence finishes the proof.

\section{REMARK 2.3}

The assumption that $x \notin \mathfrak{m}^{2}$ in Proposition 2.2(ii)(b) is necessary: Assume that $(R, \mathfrak{m})$ is a regular local ring, and assume that $0 \neq x \in \mathfrak{m}^{2}$. Set $S=R / x R$, and set $M=R$. Then $M \in \bmod R=\mathrm{T}(R)$. However, since $S$ is not regular, $M / x M \notin$ $\mathrm{T}(R / x R)$.

Recall that a local homomorphism $f: R \rightarrow S$ of local rings is called a finite local ring homomorphism if $S$ is a finitely generated $R$-module via $f$. Now we study the behavior of test modules under finite local ring homomorphisms. First, we point out that regular extensions of local rings are test modules.

\section{PROPOSITION 2.4}

Let $(R, \mathfrak{m}, k) \rightarrow(S, \mathfrak{n}, l)$ be a finite local ring homomorphism. If $S$ is regular, then $S \in \mathrm{T}(R)$.

\section{Proof}

Suppose that $\operatorname{Tor}_{\gg 0}^{R}(M, S)=0$ for some $R$-module $M$. There is an exact sequence $0 \rightarrow G_{d} \rightarrow G_{d-1} \rightarrow \cdots \rightarrow G_{0} \rightarrow S / \mathfrak{m} S \rightarrow 0$ of $S$-modules with $G_{i}$ being free. We see from this that $\operatorname{Tor}_{\gg 0}^{R}(M, S / \mathfrak{m} S)=0$. Since $S / \mathfrak{m} S=k^{(n)}$ for some $n>0$, we get $\operatorname{Tor}_{\gg 0}^{R}(M, k)=0$. This implies that $M$ has finite projective dimension, and hence $S \in \mathrm{T}(R)$.

\section{THEOREM 2.5}

Let $R \rightarrow S$ be a finite local ring homomorphism, and let $M \in \mathrm{T}(S)$. Assume that any $R$-module $X$ with $\operatorname{Tor}_{\gg 0}^{R}(S, X)=0$ satisfies $\operatorname{Tor}_{\gg 0}^{R}(M, X)=0$. Then $S \in \mathrm{T}(R)$. 
Proof

Assume on the contrary that $S \notin \mathrm{T}(R)$. Then there exists an $R$-module $L$ such that $\operatorname{Tor}_{\gg 0}^{R}(S, L)=0$ and $\operatorname{pd}_{R} L=\infty$. Hence, $\operatorname{Tor}_{>0}^{R}(S, T)=0$ for some $T=\Omega_{R}^{n} L$. Set $d=\operatorname{depth} S$, and let $N=\Omega_{R}^{d} T$. There is an exact sequence $0 \rightarrow N \rightarrow F_{d-1} \rightarrow$ $\cdots \rightarrow F_{0} \rightarrow T \rightarrow 0$ of $R$-modules, where each $F_{i}$ is a free $R$-module. We deduce that

$$
0 \rightarrow N \otimes_{R} S \rightarrow F_{d-1} \otimes_{R} S \rightarrow \cdots \rightarrow F_{0} \otimes_{R} S \rightarrow T \otimes_{R} S \rightarrow 0
$$

is exact. This implies that $\operatorname{depth}_{S}\left(N \otimes_{R} S\right) \geq d$. Using [11, A.4.20], we have isomorphisms

$$
N \otimes_{R}^{\mathbf{L}} M \simeq N \otimes_{R}^{\mathbf{L}}\left(S \otimes_{S}^{\mathbf{L}} M\right) \simeq\left(N \otimes_{R}^{\mathbf{L}} S\right) \otimes_{S}^{\mathbf{L}} M \simeq\left(N \otimes_{R} S\right) \otimes_{S}^{\mathbf{L}} M
$$

in the derived category of $R$, whose last isomorphism holds by $\operatorname{Tor}_{>0}^{R}(S, N)=0$. Thus, $\operatorname{Tor}_{i}^{R}(M, N) \cong \operatorname{Tor}_{i}^{S}\left(M, N \otimes_{R} S\right)$ for all $i>0$. By assumption, we have that $\operatorname{Tor}_{\gg 0}^{R}(M, N)=0$, whence $\operatorname{Tor}_{\gg 0}^{S}\left(M, N \otimes_{R} S\right)=0$. As $M \in \mathrm{T}(S)$, it holds that $\operatorname{pd}_{S}\left(N \otimes_{R} S\right)<\infty$. The Auslander-Buchsbaum formula shows that $N \otimes_{R} S$ is a free $S$-module. Let $G$ be a minimal free resolution of $N$ over $R$. Then $G \otimes_{R} S$ is a minimal free resolution of $N \otimes_{R} S$ over $S$. The uniqueness of minimal free resolutions implies that $G_{i} \otimes_{R} S=0$, and hence $G_{i}=0$, for all $i>0$. Therefore, $N$ is a free $R$-module, and $\operatorname{pd}_{R} L<\infty$. This is a contradiction.

We record a direct consequence of Theorem 2.5.

\section{COROLLARY 2.6}

Let $R \rightarrow S$ be a finite local ring homomorphism. If there is $M \in \mathrm{T}(S)$ with $\operatorname{pd}_{R} M<\infty$, then $S \in \mathrm{T}(R)$. In particular, if $S=R /(\underline{x})$ where $\underline{x}$ is a regular sequence on $R$, then the ring $R$ is regular.

The category of test modules over complete intersection rings is determined in terms of complexity. Recall that the complexity $\operatorname{cx}_{R} M$ of an $R$-module $M$ is the dimension of the support variety $V(M)$ associated to $M$ (see [4] and [5] for details). In an earlier version of this article, we made use of Corollary 2.6 and [23, Theorem 1.3] and proved Proposition 2.7 below for complete intersection local rings which are complete. The authors are grateful to Petter Andreas Bergh for explaining a completion-free proof of this fact.

PROPOSITION 2.7

Let $R$ be a local complete intersection. Then

$$
\mathrm{T}(R)=\left\{M \in \bmod R \mid \operatorname{cx}_{R} M=\operatorname{codim} R\right\} .
$$

Proof

(): This follows from [22, Corollary 1.2].

$(\subseteq)$ : Put $c=\operatorname{codim} R$. Let $M \in \bmod R$ with $\operatorname{cx}_{R}(M)<c$. Then $\operatorname{dim} V(M)<$ $c=\operatorname{dim} \bar{k}^{c}$, where $\bar{k}$ is the algebraic closure of $k$. This implies that there exists a 
closed homogeneous variety $W$ in $\bar{k}^{c}$ with $\operatorname{dim} W>0$ and $W \cap V(M)=\{0\}$. Now, by [7, Corollary 2.3], there exists $N \in \bmod R$ with $V(N)=W$. Since $\operatorname{cx}_{R} N=$ $\operatorname{dim} V(N)=\operatorname{dim} W>0$, the $R$-module $N$ has infinite projective dimension. Recall that $V(N) \cap V(M)=\{0\}$; thus, we deduce from [5, Theorem IV] that $\operatorname{Tor}_{\gg 0}^{R}(M$, $N)=0$. Consequently, $M \notin \mathrm{T}(R)$.

Here are some consequences of Proposition 2.7; the first one is the result of Huneke and Wiegand [20, Theorem 1.9] discussed in the introduction.

COROLLARY 2.8

(i) (Huneke-Wiegand) Let $R$ be a hypersurface. Then an $R$-module $M$ is in $\mathrm{T}(R)$ if and only if $M$ has infinite projective dimension.

(ii) Let $R \rightarrow S$ be a finite local ring homomorphism of local complete intersections. If there exists an $S$-module $M$ such that $\operatorname{cx}_{R} M=0$ and $\operatorname{cx}_{S} M=\operatorname{codim} S$, then $\operatorname{cx}_{R} S=\operatorname{codim} R$. In other words, if $M$ has minimal complexity over $R$ and has maximal complexity over $S$, then $S$ has maximal complexity over $R$.

Proof

Only the second claim in (ii) requires a proof. By Proposition 2.7, $M \in \mathrm{T}(S)$. Corollary 2.6 implies that $S \in \mathrm{T}(R)$. Again by Proposition 2.7, we have $\operatorname{cx}_{R} S=$ $\operatorname{codim} R$.

\section{REMARK 2.9}

(1) Local rings over which all modules of infinite projective dimension are test modules are not necessarily hypersurfaces. A natural example of such a ring is a Golod ring that is not Gorenstein (e.g., $\mathbb{C}\left[\left[t^{3}, t^{4}, t^{5}\right]\right]$ ).

(2) Test modules do not behave well under localization. Let $(R, \mathfrak{m})$ be a 2 dimensional local hypersurface such that $R_{p}$ is not regular for some $p \in \operatorname{Spec} R \backslash$ $\{\mathfrak{m}\}($ e.g., $R=\mathbb{C}[[x, y, z]] /(x y)$ and $p=(x, y) R)$. Let $M=\Omega_{R}^{2} k$. Then $M \in \mathrm{T}(R)$ by Corollary 2.8(i). However, $M_{p}$ is free over $R_{p}$, and hence $M_{p} \notin \mathrm{T}\left(R_{p}\right)$.

(3) Nontest modules do not behave well under localization. Let $(R, \mathfrak{m})$ be a complete intersection of codimension 2 such that $R_{p}$ is regular for all $p \in \operatorname{Spec} R \backslash$ $\{\mathfrak{m}\}$ (e.g., $\left.R=\mathbb{C}[[x, y, z, v]] /\left(x^{2}+y^{2}+z^{2}+v^{2}, x^{3}+y^{3}+z^{3}+v^{3}\right)\right)$. Let $M \in \bmod R$ with $\operatorname{cx}_{R} M=1$ (see [6, Example 5.7]). Then $M \notin \mathrm{T}(R)$ by Proposition 2.7. However, $M_{p} \in \mathrm{T}\left(R_{p}\right)$ for all $p \in \operatorname{Spec} R \backslash\{\mathfrak{m}\}$.

\section{Homological dimensions of test modules}

In this section we study the existence of test modules of finite homological dimensions. We start by recalling $\mathrm{G}_{C}$-dimension; it is a homological invariant for modules, originally introduced by Golod [16], associated to a fixed semidualizing module $C$. 


\section{DEFINITION 3.1}

Let $C$ be a semidualizing $R$-module, that is, an $R$-module $C$ such that the natural homomorphism $R \rightarrow \operatorname{Hom}_{R}(C, C)$ is an isomorphism and $\operatorname{Ext}_{R}^{>0}(C, C)=0$. An $R$-module $X$ is called totally $C$-reflexive if the natural map $X \rightarrow \operatorname{Hom}_{R}\left(\operatorname{Hom}_{R}(X\right.$, $C), C)$ is an isomorphism and $\operatorname{Ext}_{R}^{>0}(X, C)=\operatorname{Ext}_{R}^{>0}\left(\operatorname{Hom}_{R}(X, C), C\right)=0$. The $\mathrm{G}_{C}$-dimension of an $R$-module $M$, denoted by $\mathrm{G}_{C}$ - $\operatorname{dim}_{R} M$, is defined as the infimum of the integers $n \geq 0$ such that there exists an exact sequence $0 \rightarrow X_{n} \rightarrow$ $\cdots \rightarrow X_{0} \rightarrow M \rightarrow 0$, where each $X_{i}$ is totally $C$-reflexive.

A totally $R$-reflexive module is simply called totally reflexive. The $\mathrm{G}_{R}$-dimension of $M$ is nothing but the Gorenstein dimension ( $G$-dimension for short) introduced by Auslander and Bridger [3] and simply denoted by G-dim $\operatorname{dim}_{R} M$. A lot of studies on G-dimension have been done so far. The details are stated in the book [11] and the survey article [12].

Corso, Huneke, Katz, and Vasconcelos [14, Corollary 3.3] proved that integrally closed $\mathfrak{m}$-primary ideals can be used to test for finite projective dimension. More precisely, they proved that if $(R, \mathfrak{m})$ is a local ring and $I$ is an integrally closed $\mathfrak{m}$-primary ideal of $R$, then $\operatorname{Tor}_{n}^{R}(R / I, N)=0$ if and only if $\operatorname{pd}_{R} N<n$. Goto and Hayasaka [17, Theorem 1.1] proved that if such an ideal $I$ contains a non-zero-divisor of $R$ and G- $\operatorname{dim}_{R} I<\infty$, then $R$ is Gorenstein. Thus, integrally closed $\mathfrak{m}$-primary ideals are test modules, and the existence of such ideals having finite G-dimension and positive grade forces the ring to be Gorenstein.

The main purpose of this section is to generalize this. More precisely, we would like to replace the ideal $I$ considered with an arbitrary test module of finite G-dimension and deduce that $R$ is Gorenstein. For this purpose, we introduce the following category:

$$
\begin{aligned}
\operatorname{El}(R)= & \left\{M \in \bmod R \mid \text { all } R \text {-modules } N \text { with } \operatorname{Ext}_{R}^{\gg 0}(M, N)=0\right. \\
& \text { satisfy } \left.\operatorname{id}_{R} N<\infty\right\} .
\end{aligned}
$$

The theorem below is the main result of this section. We refer the reader to $[18, \mathrm{~V}],[11, \mathrm{~A} .8]$, and [24, Section 1] for details of dualizing complexes.

\section{THEOREM 3.2}

Let $R$ be a commutative Noetherian ring (not necessarily local) admitting a dualizing complex. Then one has $\mathrm{T}(R)=\mathrm{EI}(R)$.

Proof

Let $D$ be a dualizing complex of $R$. Let $M \in \mathrm{T}(R)$, and let $X \in \bmod R$ such that $\operatorname{Ext}_{R}^{\gg 0}(M, X)=0$. By [11, A.4.24] we have an isomorphism in the derived category of $R$ :

$$
M \otimes_{R}^{\mathbf{L}} Y \simeq \mathbf{R H o m}(\mathbf{R H o m}(M, X), D),
$$


where $Y:=\mathbf{R H o m}(X, D)$ is a homologically bounded complex. Since $\mathbf{R H o m}(M$, $X)$ is homologically bounded, so is $M \otimes_{R}^{\mathbf{L}} Y$. Take a projective resolution of $Y$ :

$$
(F, \partial)=\left(\cdots \rightarrow F_{i} \stackrel{\partial_{i}}{\longrightarrow} F_{i-1} \rightarrow \cdots \rightarrow F_{t+1} \stackrel{\partial_{t+1}}{\longrightarrow} F_{t} \rightarrow 0\right) .
$$

As $H_{\gg 0}(F)=0=H_{\gg 0}\left(M \otimes_{R} F\right)$, we can choose an integer $n$ such that the truncation $Q=\left(\cdots \rightarrow F_{n+1} \stackrel{\partial_{n+1}}{\longrightarrow} F_{n} \rightarrow 0\right)$ of $F$ is a projective resolution of $N:=$ coker $\partial_{n+1}$ and such that $\operatorname{Tor}_{>0}^{R}(M, N)=0$. Since $M \in \mathrm{T}(R)$, this implies that $\operatorname{pd}_{R} N<\infty$, and hence $\operatorname{pd}_{R} Q<\infty$ as $Q \simeq \Sigma^{n} N$. There is a short exact sequence of complexes (see [11, A.1.17])

$$
0 \rightarrow P \rightarrow F \rightarrow Q \rightarrow 0
$$

where $P:=\left(0 \rightarrow F_{n-1} \rightarrow \cdots \rightarrow F_{t} \rightarrow 0\right)$. Since $\operatorname{pd}_{R} P<\infty$, we have $\operatorname{pd}_{R} F<\infty$, and hence $\operatorname{pd}_{R} Y<\infty$. Thus, $\operatorname{id}_{R} \mathbf{R H o m}(Y, D)<\infty$. We have an isomorphism $X \simeq \mathbf{R H o m}(Y, D)$ by [18, V.2.1], which yields $\operatorname{id}_{R} X<\infty$. Therefore, $M \in \operatorname{El}(R)$.

Conversely, let $M \in \mathrm{EI}(R)$. Let $X \in \bmod R$ such that $\operatorname{Tor}_{\gg 0}^{R}(M, X)=0$. Similarly to the above, we can prove that $\operatorname{fd}_{R} X<\infty$; use the isomorphism $\mathbf{R H o m}\left(M \otimes_{R}^{\mathbf{L}} X, D\right) \simeq \mathbf{R H o m}(M, \mathbf{R H o m}(X, D))$ (see [11, A.4.21]). We obtain $\operatorname{pd}_{R} X<\infty$ (see [24, Remark 1.6]).

\section{COROLLARY 3.3}

Let $R$ be a commutative Noetherian ring with a dualizing complex. Assume that there exist $M \in \mathrm{T}(R)$ and $N \in \bmod R$ with $\operatorname{Supp} N=\operatorname{Spec} R$ and $\operatorname{Ext}_{R}^{\gg 0}(M, N)=0$. Then $R$ is Cohen-Macaulay. If, moreover, $\operatorname{pd}_{R} N<\infty$, then $R$ is Gorenstein.

\section{Proof}

Since $M \in \operatorname{El}(R)$ by Theorem 3.2, it holds that $\operatorname{id}_{R} N<\infty$. For all $p \in \operatorname{Spec} R$, we have $N_{p} \neq 0$ and $\operatorname{id}_{R_{p}} N_{p}<\infty$. The theorem called Bass's conjecture [9, Corollaries 9.6.2 and 9.6.4, Remark 9.6.4(iii)] yields that $R_{p}$ is Cohen-Macaulay, and so is $R$. Now assume that $\operatorname{pd}_{R} N<\infty$. Then $N_{p}$ is a nonzero $R_{p}$-module of finite projective and injective dimensions for all $p \in \operatorname{Spec} R$. Hence, $R_{p}$ is Gorenstein by [9, Exercise 3.1.25], and so is $R$.

In the next corollary, under the hypothesis that the ring considered has a dualizing complex, we obtain a generalization of the result due to Corso-HunekeKatz-Vasconcelos and Goto-Hayasaka, which accomplishes our main purpose of this section.

\section{COROLLARY 3.4}

Let $R$ be a ring with a dualizing complex, and let $M$ be a test module.

(i) If $\mathrm{G}_{C}-\operatorname{dim}_{R} M<\infty$ for some semidualizing module $C$, then $R$ is CohenMacaulay.

(ii) If $\mathrm{G}-\operatorname{dim}_{R} M<\infty$, then $R$ is Gorenstein. 
Proof

(i) Since $\operatorname{Hom}_{R}(C, C) \cong R$, we have $\operatorname{Supp} C=\operatorname{Spec} R$. It is easy to see that $\operatorname{Ext}_{R}^{\gg 0}(M, C)=0$. By Corollary $3.3, R$ is Cohen-Macaulay.

(ii) This follows from Corollary 3.3.

The conclusion of Corollary 3.4 naturally raises the following question. We refer to [6] for details of the complete intersection dimension CI- $\operatorname{dim}_{R}$.

\section{QUESTION 3.5}

Let $R$ be a local ring. Let $M$ be a test module with CI- $\operatorname{dim}_{R} M<\infty$. Then must $R$ be a complete intersection?

Corollary 2.6 more or less supports an affirmative answer to this question, but we do not know entirely. The difficulty we face here is that we do not know whether the property of being a test module is preserved under local flat extensions, even under completion.

Next we investigate the category $\operatorname{EI}(R)$ when $R$ is Cohen-Macaulay. Set

$$
\begin{aligned}
\operatorname{EP}(R)= & \left\{M \in \bmod R \mid \text { all } R \text {-modules } N \text { with } \operatorname{Ext}_{R}^{\gg 0}(N, M)=0\right. \\
& \text { satisfy } \left.\operatorname{pd}_{R} N<\infty\right\} .
\end{aligned}
$$

PROPOSITION 3.6

Let $R$ be a Cohen-Macaulay local ring with a canonical module $\omega$. Put $(-)^{\dagger}=$ $\operatorname{Hom}_{R}(-, \omega)$. For each maximal Cohen-Macaulay $R$-module $M$ one has

$$
M \in \mathrm{T}(R) \Longleftrightarrow M^{\dagger} \in \mathrm{EP}(R) .
$$

\section{Proof}

There are isomorphisms in the derived category of $R: \mathbf{R H o m}_{R}\left(X \otimes_{R}^{\mathbf{L}} M, \omega\right) \cong$ $\mathbf{R} \operatorname{Hom}_{R}\left(X, M^{\dagger}\right)$ and $\mathbf{R} \operatorname{Hom}_{R}\left(\mathbf{R H o m}_{R}\left(X, M^{\dagger}\right), \omega\right) \cong X \otimes_{R}^{\mathbf{L}} \mathbf{R} \operatorname{Hom}_{R}\left(M^{\dagger}, \omega\right) \cong$ $X \otimes_{R}^{\mathbf{L}} M$ (see [11, A.4.21 and A.4.24]). These give rise to spectral sequences

$$
\begin{aligned}
& { }^{1} E_{2}^{p, q}=\operatorname{Ext}_{R}^{p}\left(\operatorname{Tor}_{q}^{R}(X, M), \omega\right) \Rightarrow H^{p+q}=\operatorname{Ext}_{R}^{p+q}\left(X, M^{\dagger}\right) \quad \text { and } \\
& { }^{2} E_{2}^{p, q}=\operatorname{Ext}_{R}^{p}\left(\operatorname{Ext}_{R}^{-q}\left(X, M^{\dagger}\right), \omega\right) \Rightarrow H^{p+q}=\operatorname{Tor}_{-p-q}^{R}(X, M) .
\end{aligned}
$$

By using these sequences one can easily deduce the equivalence.

Recall that a local ring $R$ is called $G$-regular (see [30]) if G- $\operatorname{dim}_{R} M=\operatorname{pd}_{R} M$ for all $R$-modules $M$. The above proposition gives a sufficient condition for a local ring to be G-regular in terms of test modules.

\section{COROLLARY 3.7}

Let $R$ be a Cohen-Macaulay local ring with a canonical module $\omega$. If $\omega$ is a test module, then $R$ is G-regular. 
Proof

Proposition 3.6 implies that $R \in \operatorname{EP}(R)$. Let $M$ be an $R$-module. If $\mathrm{G}$ - $\operatorname{dim}_{R} M<$ $\infty$, then $\operatorname{Ext}_{R}^{\gg 0}(M, R)=0$, and hence $\operatorname{pd}_{R} M<\infty$. This shows that G-dim $\operatorname{dim}_{R} M=$ $\operatorname{pd}_{R} M$.

\section{Categorical approaches for nontest modules}

In this section we continue studying the homological properties of test modules, with a special attention to the full subcategory $\mathrm{NT}(R)$ of $\bmod R$ consisting of nontest modules:

$$
\begin{aligned}
\mathrm{NT}(R) & =\mathrm{T}(R)^{\mathrm{c}} \\
& =\left\{M \in \bmod R \mid \operatorname{Tor}_{\gg 0}^{R}(M, N)=0 \text { for some } R \text {-module } N \notin \mathrm{fpd}(R)\right\},
\end{aligned}
$$

where $\operatorname{fpd}(R)$ denotes the full subcategory of $\bmod R$ consisting of modules of finite projective dimension. Note that unless $R$ is regular one has

$$
\mathrm{NT}(R) \supseteq \mathrm{fpd}(R) .
$$

We begin by considering the closedness of NT $(R)$ under (finite) direct sums. First we confirm that it does not always hold.

\section{EXAMPLE 4.1}

Let $k$ be a field, and put $R=k[x, y, z] /\left(x^{2}, y^{2}, z^{2}, y z\right)$. Then $R$ is a non-Gorenstein local ring such that the cube of the maximal ideal is zero. Let $M=R /(x)$, and let $N=R /(y, z)$. Then $M, N \notin \mathrm{fpd}(R)$ and $\operatorname{Tor}_{>0}^{R}(M, N)=0$; hence, $M, N \in$ $\mathrm{NT}(R)$. Suppose that $\operatorname{Tor}_{\gg 0}^{R}(M \oplus N, L)=0$ for some $L \in \bmod R$. There are exact sequences $0 \rightarrow k^{2} \rightarrow M \rightarrow k \rightarrow 0$ and $0 \rightarrow k \rightarrow N \rightarrow k \rightarrow 0$. The first sequence implies that $\operatorname{Tor}_{i+1}^{R}(k, L) \cong \operatorname{Tor}_{i}^{R}\left(k^{2}, L\right)$, and hence $\beta_{i+1}^{R}(L)=2 \beta_{i}^{R}(L)$ for $i \gg 0$. Similarly, it follows from the second sequence that $\beta_{i+1}^{R}(L)=\beta_{i}^{R}(L)$ for $i \gg 0$. Such equalities of Betti numbers of $L$ can occur only when $\operatorname{pd}_{R} L<\infty$. This proves that $M \oplus N$ is a test module, that is, that $M \oplus N \notin \mathrm{NT}(R)$.

In general we have the following result.

\section{PROPOSITION 4.2}

Let $(R, \mathfrak{m}, k)$ be a non-Gorenstein local ring with $\mathfrak{m}^{3}=0$. Let $M$ be a nonfree totally reflexive $R$-module. Then $M, E_{R}(k) \in \mathrm{NT}(R)$ and $M \oplus E_{R}(k) \notin \mathrm{NT}(R)$.

\section{Proof}

Note that $M$ and $E:=E_{R}(k)$ have infinite projective dimension. Corollary 3.7 implies that $E \in \mathrm{NT}(R)$. Setting $(-)^{*}=\operatorname{Hom}(-, R)$ and $(-)^{\vee}=\operatorname{Hom}(-, E)$, we deduce that

$$
M \otimes_{R}^{\mathbf{L}} E \cong M \otimes_{R}^{\mathbf{L}} \mathbf{R H o m}_{R}(R, E) \cong \mathbf{R} \operatorname{Hom}_{R}\left(\mathbf{R H o m}_{R}(M, R), E\right) \cong M^{* \vee} .
$$


Here the second isomorphism follows from [11, A.4.24], and the total reflexivity of $M$ yields the third isomorphism. Hence, we have $\operatorname{Tor}_{>0}^{R}(M, E)=0$. In particular, $M, E \in \mathrm{NT}(R)$.

Let $L \in \bmod R$, and assume that $\operatorname{Tor}_{\gg 0}^{R}(M \oplus E, L)=0$. Then $\operatorname{Tor}_{\gg 0}^{R}(M, L)=$ $\operatorname{Tor}_{\gg 0}^{R}(E, L)=0$. It follows from [19, Proposition 2.9] that $\operatorname{cx}_{R} L \leq 1$ and $\operatorname{cx}_{R} M=1$. One also sees that $\operatorname{cx}_{R}\left(M \otimes_{R} L\right)=\operatorname{cx}_{R} M+\operatorname{cx}_{R} L$. The complexity of an $R$-module can only be 0,1 , or $\infty$ by [26] (see also [13, 1.1]). Hence, $\mathrm{cx}_{R} L=0$; that is, $\operatorname{pd}_{R} L<\infty$. Thus, $M \oplus E \in \mathrm{T}(R)$.

In fact, closure under direct sums is equivalent to closure under extensions.

\section{PROPOSITION 4.3}

Let $R$ be a local ring. The following are equivalent:

(i) $\mathrm{NT}(R)$ is closed under extensions.

(ii) $\mathrm{NT}(R)$ is closed under direct sums.

Proof

Clearly, (i) implies (ii). Assume that (ii) holds. Let $0 \rightarrow M \rightarrow U \rightarrow N \rightarrow 0$ be an exact sequence with $M, N \in \mathrm{NT}(R)$. Then there is an $R$-module $X$ with $\operatorname{pd}_{R} X=$ $\infty$ such that $\operatorname{Tor}_{\gg 0}^{R}(M \oplus N, X)=0$. Hence, $\operatorname{Tor}_{\gg 0}^{R}(U, X)=0$, and thus $U \in$ $\mathrm{NT}(R)$.

If $R$ is a nonregular complete intersection, then $\mathrm{NT}(R)$ is closed under direct sums by Proposition 2.7. Hence, we deduce the following result from Proposition 4.3.

\section{COROLLARY 4.4}

If $\mathrm{NT}(R)$ is closed under direct sums, then $\mathrm{NT}(R)$ is a resolving subcategory of $\bmod R$. Thus, $\mathrm{NT}(R)$ is resolving when $R$ is a nonregular complete intersection.

We state here a conjecture of David A. Jorgensen (personal communication, 2011) (cf. Remark 4.8 below).

\section{CONJECTURE 4.5}

Let $R$ be a local ring. Assume that $\mathrm{NT}(R) \neq \mathrm{fpd}(R)$. If $\mathrm{NT}(R)$ is closed under direct sums, then $R$ is a complete intersection of codimension at least 2 .

Next we investigate nontest modules in resolving subcategories. For $M \in \bmod R$ we denote by res $M$ the resolving closure of $M$. The full subcategory of $\bmod R$ consisting of maximal Cohen-Macaulay modules is denoted by $\mathrm{CM}(R)$.

PROPOSITION 4.6

Let $R$ be a Henselian local ring, and let $\mathcal{X}$ be a resolving subcategory of $\bmod R$. Suppose that there are only finitely many nonisomorphic indecomposable modules 
in $\mathcal{X} \cap \mathrm{NT}(R)$. Then $\operatorname{Ext}_{R}^{\gg 0}(M, R)=0$ if and only if $\operatorname{pd}_{R} M<\infty$ for all $M \in$ $\mathcal{X} \cap \mathrm{NT}(R)$. In particular, $\mathrm{G}-\operatorname{dim}_{R} M=\operatorname{pd}_{R} M$.

Proof

Take a module $M \in \mathcal{X} \cap \mathrm{NT}(R)$ with $\operatorname{Ext}_{R}^{\gg 0}(M, R)=0$. Assume that $\operatorname{pd}_{R} M=\infty$. Given $X \in \bmod R$, we set $\mathrm{NT}_{X}(R)=\left\{M \in \bmod R \mid \operatorname{Tor}_{\gg 0}^{R}(M, X)=0\right\}$. It is easy to see that $\mathrm{NT}_{X}(R)$ is a resolving subcategory of $\bmod R$, and

$$
\mathrm{NT}(R)=\bigcup_{\operatorname{pd} X=\infty} \mathrm{NT}_{X}(R) .
$$

There is an $R$-module $X$ with $\operatorname{pd}_{R} X=\infty$ and $M \in \mathrm{NT}_{X}(R)$. Hence, res $M \subseteq$ $\mathrm{NT}_{X}(R) \subseteq \mathrm{NT}(R)$, and we have res $M \subseteq \mathcal{X} \cap \mathrm{NT}(R)$. By assumption, there are only finitely many nonisomorphic indecomposable modules in res $M$ (so res $M$ is contravariantly finite). As $\Omega^{d} k \in \mathrm{T}(R)$, we have $\Omega^{d} k \notin$ res $M$. Hence, by [32, Theorem 1.4], $R$ is Cohen-Macaulay and res $M=\mathrm{CM}(R)$. This is a contradiction since $\Omega^{d} k \in \mathrm{CM}(R)$. Consequently, $\operatorname{pd}_{R} M<\infty$.

\section{COROLLARY 4.7}

Let $R$ be a Henselian local ring. Assume that there are only finitely many indecomposable $R$-modules (up to isomorphism) in $\mathrm{CM}(R) \cap \mathrm{NT}(R)$.

(i) If $M \in \mathrm{CM}(R) \cap \mathrm{NT}(R)$, then either $M$ is free or $\mathrm{G}-\operatorname{dim}_{R} M=\infty$.

(ii) If $R$ is Gorenstein and nonregular, then $\mathrm{NT}(R)=\mathrm{fpd}(R)$.

\section{Proof}

The assertion (i) is immediate from Proposition 4.6: take $\mathcal{X}=\mathrm{CM}(R)$. As to (ii), let $Z \in \mathrm{NT}(R)$. Then $\Omega^{d} Z \in \mathrm{CM}(R) \cap \mathrm{NT}(R)$, where $d=\operatorname{dim} R$. By (i), $\Omega^{d} Z$ is free.

\section{REMARK 4.8}

Recall that $\mathrm{NT}(R)=\mathrm{fpd}(R)$ if $R$ is a hypersurface (Corollary 2.8(i)). In view of this fact, it is worth noting that a Henselian Gorenstein ring satisfying the hypotheses of Corollary 4.7 is not necessarily a hypersurface: Let $k$ be a field, and let $R=k[x, y, z] /\left(x^{2}-y^{2}, x^{2}-z^{2}, x y, x z, y z\right)$. Then $R$ is an Artinian (hence, Henselian) Gorenstein local ring that is not a hypersurface. By [19, Theorem 3.1(2)] we see that $\mathrm{NT}(R)=\mathrm{fpd}(R)$ and that $R$ is the unique indecomposable module in $\mathrm{CM}(R) \cap \mathrm{NT}(R)$.

It is not known whether there exist modules $M$ over arbitrary local rings $R$ such that $\operatorname{pd} M=\infty$ and $\operatorname{Tor}_{\gg 0}^{R}(M, M)=0$. Our next result determines certain conditions, in terms of the category $\mathrm{NT}(R)$, for the existence of such modules.

\section{PROPOSITION 4.9}

Let $R$ be a local ring. Assume that $\mathrm{NT}(R) \neq \mathrm{fpd}(R)$, and assume that $\mathrm{NT}(R)=$ 
res $M$ for some $M \in \bmod R$. Then there is $X \in \mathrm{NT}(R)$ with $\operatorname{pd}_{R} X=\infty$ and $\operatorname{Tor}_{\gg 0}^{R}(X, X)=0$.

Proof

If $\operatorname{pd}_{R} M<\infty$, then res $M \subseteq \mathrm{fpd}(R)$; hence, $\mathrm{NT}(R)=\mathrm{fpd}(R)$. This contradiction shows $\operatorname{pd}_{R} M=\infty$. Since $M \in \mathrm{NT}(R)$, we have $M \in \mathrm{NT}_{X}(R)$ for some module $X$ with $\operatorname{pd}_{R} X=\infty$. As $\operatorname{pd}_{R} M=\infty$, we see that $X \in \mathrm{NT}(R)$. It holds that $\mathrm{NT}(R)=\operatorname{res} M \subseteq \mathrm{NT}_{X}(R)$, whence $\mathrm{NT}(R)=\mathrm{NT}_{X}(R)$. Thus, $X \in \mathrm{NT}_{X}(R)$, so that $\operatorname{Tor}_{\gg 0}^{R}(X, X)=0$.

Using the above proposition, we obtain an interesting property of $\mathrm{NT}(R)$.

COROLLARY 4.10

Let $R$ be a local complete intersection of codimension at least 2 . Then $\mathrm{NT}(R) \neq$ res $M$ for all $R$-modules $M$.

Proof

It follows from [6, Example 5.7] that there is an $R$-module $N$ of complexity 1. By Proposition 2.7, $N$ is a nontest module. Therefore, if $\operatorname{NT}(R)=\operatorname{res} M$ for some $M \in \bmod R$, then Proposition 4.9 implies that there exists $X \in \mathrm{NT}(R)$ with $\operatorname{pd}_{R} X=\infty$ and $\operatorname{Tor}_{\gg 0}^{R}(X, X)=0$. Since $R$ is a complete intersection, such an $R$-module $X$ cannot exist by [22, Corollary 1.2].

\section{REMARK 4.11}

The assumption of Corollary 4.10 on the codimension cannot be weakened. Indeed, let $R$ be a hypersurface (i.e., $\operatorname{codim} R \leq 1)$. Then $\operatorname{NT}(R)=\mathrm{fpd}(R)$ by Corollary 2.8(i). So, if $R$ is reduced of dimension 1 , then $\mathrm{NT}(R)$ coincides with the resolving closure of the Auslander transpose of $k$ by [15, Theorem 2.1].

Acknowledgments. This work started around "Homological Days" held at the University of Kansas in May, 2011. We would like to thank the Department of Mathematics of the University of Kansas for hosting the conference. Our special thanks are due to Petter Andreas Bergh and David A. Jorgensen for valuable comments and suggestions on earlier versions of this paper. Part of this work was done during the visit of the third author to University of Missouri in November 2011 and the visit of the first author to University of Kansas in April 2012. We are grateful for the kind hospitality of both institutions. Finally, we thank the referee for valuable comments.

\section{References}

[1] T. Araya, K.-I. Iima, and R. Takahashi, On the left perpendicular category of the modules of finite projective dimension, Comm. Algebra 40 (2012), 2693-2699. MR 2968905. DOI 10.1080/00927872.2011.584926. 
[2] J. Asadollahi and T. J. Puthenpurakal, "An analogue of a theorem due to Levin and Vasconcelos" in Commutative Algebra and Algebraic Geometry, Contemp. Math. 390, Amer. Math. Soc., Providence, 2005, 9-15. MR 2187321. DOI 10.1090/conm/390/07290.

[3] M. Auslander and M. Bridger, Stable Module Theory, Mem. Amer. Math. Soc. 94, Amer. Math. Soc., Providence, 1969. MR 0269685.

[4] L. L. Avramov, "Infinite free resolution" in Six Lectures on Commutative Algebra (Bellaterra, 1996), Progr. Math. 166, Birkhäuser, Basel, 1998, 1-118. MR 1648664.

[5] L. L. Avramov and R.-O. Buchweitz, Support varieties and cohomology over complete intersections, Invent. Math. 142 (2000), 285-318. MR 1794064. DOI $10.1007 / \mathrm{s} 002220000090$.

[6] L. L. Avramov, V. N. Gasharov, and I. V. Peeva, Complete intersection dimension, Inst. Hautes Études Sci. Publ. Math. 86 (1997), 67-114. MR 1608565.

[7] P. A. Bergh, On support varieties for modules over complete intersections, Proc. Amer. Math. Soc. 135 (2007), 3795-3803. MR 2341929. DOI 10.1090/S0002-9939-07-09009-0.

[8] _ On the vanishing of homology for modules of finite length, Math. Scand. 112 (2013), 11-18. MR 3057596.

[9] W. Bruns and J. Herzog, Cohen-Macaulay Rings, rev. ed., Cambridge Stud. Adv. Math. 39, Cambridge Univ. Press, Cambridge, 1998. MR 1251956.

[10] O. Celikbas and H. Dao, Asymptotic behavior of Ext functors for modules of finite complete intersection dimension, Math. Z. 269 (2011), 1005-1020. MR 2860275. DOI 10.1007/s00209-010-0771-9.

[11] L. W. Christensen, Gorenstein Dimensions, Lecture Notes in Math. 1747, Springer, Berlin, 2000. MR 1799866. DOI 10.1007/BFb0103980.

[12] L. W. Christensen, H.-B. Foxby, and H. Holm, "Beyond totally reflexive modules and back: A survey on Gorenstein dimensions" in Commutative Algebra-Noetherian and Non-Noetherian Perspectives, Springer, New York, 2011, 101-143. MR 2762509. DOI 10.1007/978-1-4419-6990-3_5.

[13] L. W. Christensen and O. Veliche, Acyclicity over local rings with radical cube zero, Illinois J. Math. 51 (2007), 1439-1454. MR 2417436.

[14] A. Corso, C. Huneke, D. Katz, and W. V. Vasconcelos, "Integral closure of ideals and annihilators of homology" in Commutative Algebra, Lect. Notes Pure Appl. Math. 244, Chapman and Hall/CRC, Boca Raton, Fla., 2006, 33-48. MR 2184788. DOI 10.1201/9781420028324.ch4.

[15] H. Dao and R. Takahashi, Classification of resolving subcategories and grade consistent functions, to appear in Int. Math. Res. Not. IMRN (2013). DOI 10.1093/imrn/rnt141.

[16] E. S. Golod, G-dimension and generalized perfect ideals, Trudy Mat. Inst. Steklov. 165 (1984), 62-66. MR 0752933. 
[17] S. Goto and F. Hayasaka, Finite homological dimension and primes associated to integrally closed ideals, Proc. Amer. Math. Soc. 130 (2002), 3159-3164. MR 1912992. DOI 10.1090/S0002-9939-02-06436-5.

[18] R. Hartshorne, Residues and Duality, with an appendix by P. Deligne, Lecture Notes in Math. 20, Springer, Berlin, 1966. MR 0222093.

[19] C. Huneke, L. M. Şega, and A. N. Vraciu, Vanishing of Ext and Tor over some Cohen-Macaulay local rings, Illinois J. Math. 48 (2004), 295-317. MR 2048226.

[20] C. Huneke and R. Wiegand, Tensor products of modules, rigidity and local cohomology, Math. Scand. 81 (1997), 161-183. MR 1612887.

[21] S. Iyengar and T. J. Puthenpurakal, Hilbert-Samuel functions of modules over Cohen-Macaulay rings, Proc. Amer. Math. Soc. 135 (2007), 637-648. MR 2262858. DOI 10.1090/S0002-9939-06-08519-4.

[22] D. A. Jorgensen, Tor and torsion on a complete intersection, J. Algebra 195 (1997), 526-537. MR 1469637. DOI 10.1006/jabr.1997.7064.

[23] Complexity and Tor on a complete intersection, J. Algebra 211 (1999), 578-598. MR 1666660. DOI 10.1006/jabr.1998.7743.

[24] P. Jørgensen, Existence of Gorenstein projective resolutions and Tate cohomology, J. Eur. Math. Soc. (JEMS) 9 (2007), 59-76. MR 2283103. DOI 10.4171/JEMS/72.

[25] P. Jothilingam, Test modules for projectivity, Proc. Amer. Math. Soc. 94 (1985), 593-596. MR 0792267. DOI 10.2307/2044870.

[26] J. Lescot, Asymptotic properties of Betti numbers of modules over certain rings, J. Pure Appl. Algebra 38 (1985), 287-298. MR 0814184. DOI 10.1016/0022-4049(85)90016-7.

[27] G. Levin and W. V. Vasconcelos, Homological dimensions and Macaulay rings, Pacific J. Math. 25 (1968), 315-323. MR 0230715.

[28] C. Miller, Complexity of tensor products of modules and a theorem of Huneke-Wiegand, Proc. Amer. Math. Soc. 126 (1998), 53-60. MR 1415354. DOI 10.1090/S0002-9939-98-04017-9.

[29] M. Ramras, On the vanishing of Ext, Proc. Amer. Math. Soc. 27 (1971), 457-462. MR 0284427.

[30] R. Takahashi, On G-regular local rings, Comm. Algebra 36 (2008), 4472-4491. MR 2473342. DOI 10.1080/00927870802179602.

[31] Classifying thick subcategories of the stable category of Cohen-Macaulay modules, Adv. Math. 225 (2010), 2076-2116. MR 2680200. DOI 10.1016/j.aim.2010.04.009.

[32] Contravariantly finite resolving subcategories over commutative rings, Amer. J. Math. 133 (2011), 417-436. MR 2797352. DOI 10.1353/ajm.2011.0011.

[33] A. Tehranian, M. Tousi, and S. Yassemi, Characterizing local rings via test modules, Comm. Algebra 35 (2007), 2524-2532. MR 2345798. DOI 10.1080/00927870701326411. 
Celikbas: Department of Mathematics, University of Missouri-Columbia, 323 Mathematical Sciences Building, Columbia, Missouri 65211, USA;

celikbaso@missouri.edu; http://www.math.missouri.edu/ celikbaso/

Dao: Department of Mathematics, University of Kansas, 1460 Jayhawk Boulevard, 405 Snow Hall, Lawrence, Kansas 66045-7523, USA; hdao@math.ku.edu; http://www.math.ku.edu/ hdao/

Takahashi: Graduate School of Mathematics, Nagoya University, Furocho, Chikusaku, Nagoya 464-8602, Japan; and

Department of Mathematics, University of Nebraska, Lincoln, Nebraska 68588-0130, USA; takahashi@math.nagoya-u.ac.jp; http://www.math.nagoya-u.ac.jp/ takahashi/ 